\title{
Learning Aerial Image Segmentation from Online Maps
}

\author{
Pascal Kaiser, Jan Dirk Wegner, Aurélien Lucchi, Martin Jaggi, Thomas Hofmann, and Konrad Schindler
}

\begin{abstract}
This study deals with semantic segmentation of high-resolution (aerial) images where a semantic class label is assigned to each pixel via supervised classification as a basis for automatic map generation. Recently, deep convolutional neural networks (CNNs) have shown impressive performance and have quickly become the de-facto standard for semantic segmentation, with the added benefit that task-specific feature design is no longer necessary. However, a major downside of deep learning methods is that they are extremely data-hungry, thus aggravating the perennial bottleneck of supervised classification, to obtain enough annotated training data. On the other hand, it has been observed that they are rather robust against noise in the training labels. This opens up the intriguing possibility to avoid annotating huge amounts of training data, and instead train the classifier from existing legacy data or crowd-sourced maps which can exhibit high levels of noise. The question addressed in this paper is: can training with large-scale, publicly available labels replace a substantial part of the manual labeling effort and still achieve sufficient performance? Such data will inevitably contain a significant portion of errors, but in return virtually unlimited quantities of it are available in larger parts of the world. We adapt a state-of-the-art CNN architecture for semantic segmentation of buildings and roads in aerial images, and compare its performance when using different training data sets, ranging from manually labeled, pixel-accurate ground truth of the same city to automatic training data derived from OpenStreetMap data from distant locations. We report our results that indicate that satisfying performance can be obtained with significantly less manual annotation effort, by exploiting noisy large-scale training data.
\end{abstract}

\section{INTRODUCTION}

$\mathbf{H}$ UGE volumes of optical overhead imagery are captured every day with airborne or spaceborne platforms, and that volume is still growing. This "data deluge" makes manual interpretation prohibitive, hence machine vision must be employed if we want to make any use of the available data. Perhaps the fundamental step of automatic mapping is to assign a semantic class to each pixel, i.e. convert the raw data to a semantically meaningful raster map (which can then be further processed as appropriate with, e.g., vectorisation or map generalisation techniques). The most popular tool for that task is supervised machine learning. Supervision with human-annotated training data is necessary to inject the taskspecific class definitions into the generic statistical analysis. In most cases, reference data for classifier training is generated manually for each new project, which is a time-consuming and costly process. Manual annotation must be repeated every time the task, the geographic location, the sensor characteristics or the imaging conditions change, hence the process scales poorly. In this paper, we explore the trade-off between:

All authors are with ETH Zurich, 8093 Zurich, Switzerland
- pixel-accurate

but small-scale ground truth available; and

- less accurate reference data that is readily available in arbitrary quantities, at no cost.

For our study, we make use of online map data from OpenStreetMap [1-3] (OSM, http://www.openstreetmap.org) to automatically derive weakly labeled training data for three classes, buildings, roads, and background (i.e. all others). This data is typically collected using two main sources: (i) volunteers collect OSM data either in situ with GPS trackers or by manually digitizing very high-resolution aerial or satellite images that have been donated, and (ii) national mapping agencies donate their data to OSM to make it available to a wider public. Since OSM is generated by volunteers, our approach can be seen as a form of crowd-sourced data annotation; but other existing map databases, e.g. legacy data within a mapping agency, could also be used.

As image data for our study, we employ high-resolution RGB orthophotos from Google Map $\sqrt{1}$ since we could not easily get access to comparable amounts of other highresolution imagery $\left(>100 \mathrm{~km}^{2}\right.$ at $\approx 10 \mathrm{~cm}$ ground sampling distance (GSD)).

Clearly, this type of training data will be less accurate. Sources of errors include co-registration errors, e.g., in our case OSM polygons and Google images were independently geo-referenced; limitations of the data format, e.g., OSM only has road centerlines and category, but no road boundaries; temporal changes not depicted in outdated map or image data; or simply sloppy annotations, not only because of a lack of training or motivation, but also because the use cases of most OSM users require not even meter-level accuracy.

Our study is driven by the following hypotheses:

- The sheer volume of training data can possibly compensate for the lower accuracy (if used with an appropriate, robust learning method).

- The large variety present in very large training sets (e.g., spanning multiple different cities) could potentially improve the classifier's ability to generalise to new, unseen locations.

- Even if high-quality training data is available, the large volume of additional training data could potentially improve the classification.

- If low-accuracy, large-scale training data helps, then it may also allow one to substitute a large portion of the manually annotated high-quality data.

${ }^{1}$ specifications of Google Maps data can be found at https://support.google. com/mapcontentpartners/answer/144284?hl=en 
We investigate these hypotheses when using deep convolutional neural networks (CNNs). Deep networks are at present the top-performing method for high-resolution semantic labelling and are therefore the most appropriate choice for our study 2 At the same time they also fulfil the other requirements for our study: they are data-hungry and robust to label noise [4]. And they make manual feature design somewhat obsolete: once training data is available, retraining for different sensor types or imaging conditions is fully automatic, without scene-specific user interaction such as feature definition or preprocessing. We adopt a variant of the fully convolution network (FCN) [5], and explore the potential of combining end-to-end trained deep networks with massive amounts of noisy OSM labels. We evaluate the extreme variant of our approach, without any manual labelling, on three major cities (Chicago, Paris, Zurich) with different urban structures. Since quantitative evaluations on these large datasets are limited by the inaccuracy of the labels, which is also present in the test sets, we also perform experiments for a smaller dataset from the city of Potsdam. There, high-precision manually annotated ground truth is available, which allows us to compare different levels of project-specific input, including the baseline where only manually labelled training data is used, the extreme case of only automatically generated training labels, and variants in between. We also assess the models' capabilities regarding generalisation and transfer learning between unseen geographic locations.

We find in this study that training on noisy labels does work well, but only with substantially larger training sets. Whereas with small training sets $\left(\approx 2 \mathrm{~km}^{2}\right)$ it does not reach the performance of hand-labelled, pixel-accurate training data. Moreover, even in the presence of high-quality training data, massive OSM labels further improve the classifier, and hence can be used to significantly reduce the manual labelling efforts. According to our experiments, the differences are really due to the training labels, since segmentation performance of OSM labels is stable across different image sets of the same scene.

For practical reasons, our study is limited to buildings and roads, which are available from OSM; and to RGB images from Google Maps, subject to unknown radiometric manipulations. We hope that similar studies will also be performed with the vast archives of proprietary image and map data held by state mapping authorities and commercial satellite providers. Finally, this is a step in a journey that this will ultimately bring us closer to the utopian vision that a whole range of mapping tasks no longer need user input, but can be completely automated by the world wide web.

\section{RELATED WORK}

There is a huge literature about semantic segmentation in remote sensing. A large part deals with rather low-resolution satellite images, whereas our work in this paper deals with very high-resolution aerial images (see [6] for an overview).

\footnotetext{
${ }^{2}$ All top-performing methods on big benchmarks are CNN variants, both in generic computer vision, e.g., the Pascal VOC Challenge, http://host. robots.ox.ac.uk/pascal/VOC/ and in remote sensing, e.g., the ISPRS semantic labeling challenge, http://www2.isprs.org/commissions/comm3/wg4/ semantic-labeling.html
}

Aerial data with a ground sampling distance $G S D \leq 20 \mathrm{~cm}$ contains rich details about urban objects such as roads, buildings, trees, and cars, and is a standard source for urban mapping projects. Since urban environments are designed by humans according to relatively stable design constraints, early work attempted to construct object descriptors via sets of rules, most prominently for building detection in 2D [7, 8] or in 3D [9-11], and for road extraction [12-14]. A general limitation of hierarchical rule systems, be they top-down or bottom-up, is poor generalization across different city layouts. Hard thresholds at early stages tend to delete information that can hardly be recovered later, and hard-coded expert knowledge often misses important evidence that is less obvious to the human observer.

Machine learning thus aims to learn classification rules directly from the data. As local evidence, conventional classifiers are fed with raw pixel intensities, simple arithmetic combinations such as vegetation indices, and different statistics or filter responses that describe the local image texture [15. 17]. An alternative is to pre-compute a large, redundant set of local features for training and let a discriminative classifier (e.g., boosting, random forest) select the optimal subset [1821] for the task.

More global object knowledge that cannot be learned from local pixel features can be introduced via probabilistic priors. Two related probabilistic frameworks have been successfully applied to this task, Marked Point Processes (MPP) and graphical models. For example, [22, 23] formulate MPPs that explicitly model road network topologies while [24] use a similar approach to extract building footprints. MPPs rely on object primitives like lines or rectangles that are matched to the image data by sampling. Even if data-driven [25], such Monte-Carlo sampling has high computational cost and does not always find good configurations. Graphical models provide similar modeling flexibility, but in general also lead to hard optimization problems. For restricted cases (e.g., submodular objective functions) efficient optimisers exist. Although there is a large body of literature that aims to tailor conditional random fields (CRF) for object extraction in computer vision and remote sensing, relatively few authors tackle semantic segmentation in urban scenes [e.g. 26-30].

Given the difficulty of modeling high-level correlations, much effort has gone into improving the local evidence by finding more discriminative object features [21, 31, 32]. The resulting feature vectors are fed to a standard classifier (e.g., Decision Trees or Support Vector Machines) to infer probabilities per object category. Some authors invest a lot of effort to reduce the dimension of the feature space to a maximally discriminative subset [e.g. 33-36], although this seems to have only limited effect - at least with modern discriminative classifiers.

Deep neural networks do not require a separate feature definition step, but instead learn the most discriminative feature set for a given dataset and task directly from raw images. They go back to [37, 38], but at the time were limited by a lack of compute power and training data. After their comeback in the 2012 ImageNet challenge [39, 40], deep learning approaches, and in particular deep convolutional neural networks (CNNs), 
have achieved impressive results for diverse image analysis tasks. State-of-the-art network architectures [e.g., 41] have many (often 10-20, but up to $>100$ ) layers of local filters and thus large receptive fields in the deep layers, which makes it possible to learn complex local-to-global (non-linear) object representations and long-range contextual relations directly from raw image data. An important property of deep convolutional neural networks (CNN) is that both training and inference are easily parallelizable, especially on GPUs, and thus scale to millions of training and testing images.

Quickly, CNNs were also applied to semantic segmentation of images [42]. Our approach in this paper is based on the fully convolutional network (FCN) architecture of [5], which returns a structured, spatially explicit label image (rather than a global image label). While spatial aggregation is nevertheless required to represent context, FCNs also include in-network upsampling back to the resolution of the original image. They have already been successfully applied to semantic segmentation of aerial images, [e.g., 43-45]. In fact, the top performers on the ISPRS semantic segmentation benchmark all use CNNs. We note that (non-convolutional) deep networks in conjunction with OSM labels have also been applied for patch-based road extraction in overhead images of $\approx 1 \mathrm{~m}$ GSD at large scale [46, 47]. More recently, [48] combine OpenStreetMap (OSM) data with aerial images to augment maps with additional information from imagery like road widths. They design a sophisticated random field to probabilistically combine various sources of road evidence, for instance cars, to estimate road widths at global scale using OSM and aerial images.

To the best of our knowledge, only two works have made attempts to investigate how results of CNNs trained on largescale OSM labels can be fine-tuned to achieve more accurate results for labeling remote sensing images [49, 50]. However, we are not aware of any large-scale, systematic, comparative and quantitative study that investigates using large-scale training labels from inaccurate map data for semantic segmentation of aerial images.

\section{METHODS}

We first describe our straight-forward approach to generate training data automatically from OSM, and then give technical details about the employed FCN architecture and the training procedure used to train our model.

\section{A. Generation of Training Data}

We use a simple, automatic approach to generate datasets of very-high resolution (VHR) aerial images in RGB format and corresponding labels for classes building, road, and background. Aerial images are downloaded from Google Maps and geographic coordinates of buildings and roads are downloaded from OSM. We prefer to use OSM maps instead of Google Maps, because the latter can only be downloaded as raster images ${ }^{3}$ OSM data can be accessed and manipulated in vector

\footnotetext{
${ }^{3}$ Note that some national mapping agencies also provide publicly available map and other geo-data, e.g. the USGS national map program: https: //nationalmap.gov/
}

format, each object type comes with meta data and identifiers that allow straight-forward filtering. Regarding co-registration, we find that OSM and Google Maps align relatively well, even though they have been acquired and processed separately $4^{4}$ Most local misalignments are caused by facades of high buildings that overlap with roads or background due to perspective effects. It is apparent that in our test areas Google provides ortho-photos rectified w.r.t. a bare earth digital terrain model (DTM), not "true" ortho-photos rectified with a digital surface model (DSM). According to our own measurements on a subset of the data, this effect is relatively mild, generally $<10$ pixels displacement. We found that this does not introduce major errors as long as there are no high-rise buildings. It may be more problematic for extreme scenes such as Singapore or Manhattan.

To generate pixel-wise label maps, the geographic coordinates of OSM building corners and road center-lines are transformed to pixel coordinates. For each building, a polygon through the corner points is plotted at the corresponding image location. For roads the situation is slightly more complex. OSM only provides coordinates of road center-lines, but no precise road widths. There is, however, a road category label ("highway tag") for most roads. We determined an average road width for each category on a small subset of the data, and validated it on a larger subset (manually, one-off). This simple strategy works reasonably well, with a mean error of $\approx 11$ pixels for the road boundary, compared to $\approx 100$ pixels of road width ${ }^{5}$ In (very rare) cases where the ad-hoc procedure produced label collisions, pixels claimed by both building and road were assigned to buildings. Pixels neither labeled building nor road form the background class. Examples of images overlaid with automatically generated OSM labels are shown in Fig 1 .

\section{B. Neural network architecture}

We use a variant of fully convolutional networks (FCN) in this paper, see Fig. 2. Following the standard neural network concept, transformations are ordered in sequential layers that gradually transform the pixel values to label probabilities. Most layers implement learned convolution filters, where each neuron at level $l$ takes its input values only from a fixed-size, spatially localized window $\mathcal{W}$ in the previous layer $(l-1)$, and outputs a vector of differently weighted sums of those values, $c^{l}=\sum_{i \in \mathcal{W}} w_{i} c_{i}^{l-1}$. Weights $w_{i}$ are shared across all neurons of a layer, which reflects the shift-invariance of the image signal and drastically reduces the number of parameters. Each convolutional layer is followed by a rectified linear unit $(\operatorname{Re} L U) c_{\text {rec }}^{l}=\max \left(0, c^{l}\right)$, which simply truncates all negative values to 0 and leaves positive values unchanged [51 $]^{6}$ Convolutional layers are interspersed with Max-Pooling layers that downsample the image and retain only the maximum value

\footnotetext{
${ }^{4}$ Note that it is technically possible to obtain world coordinates of objects in Google Maps and enter those into OSM, and this might in practice also be done to some extent. However, OSM explicitly asks users not to do that.

${ }^{5}$ Average deviation based on 10 random samples of Potsdam, Chicago, Paris, and Zurich.

${ }^{6}$ Other non-linearities are sometimes used, but $R e L U$ has been shown to facilitate training (backpropagation) and has become the de-facto standard.
} 


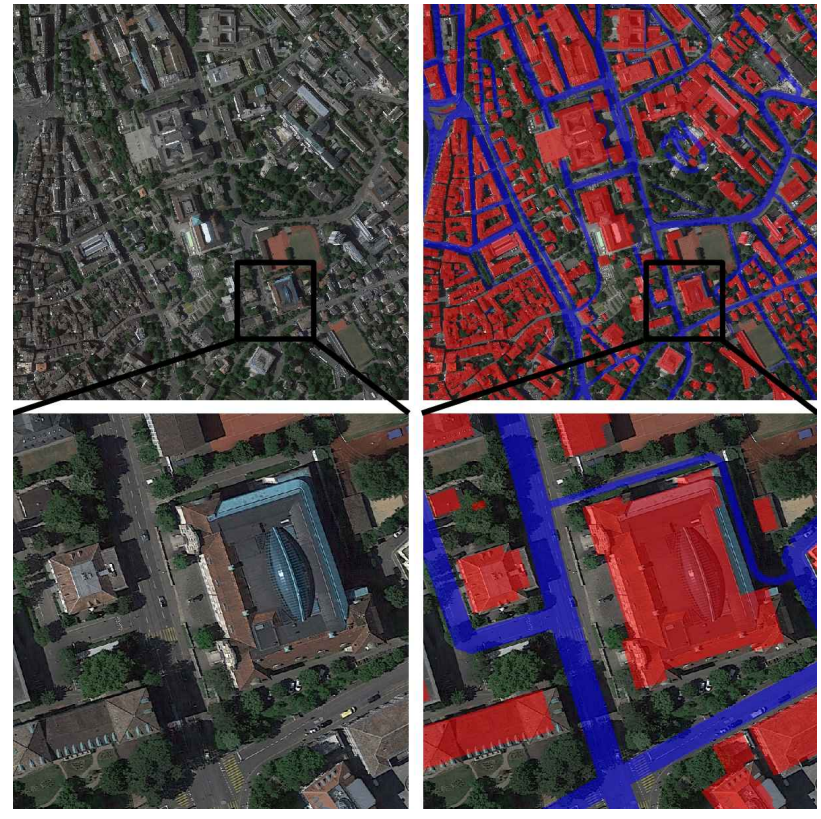

(a)
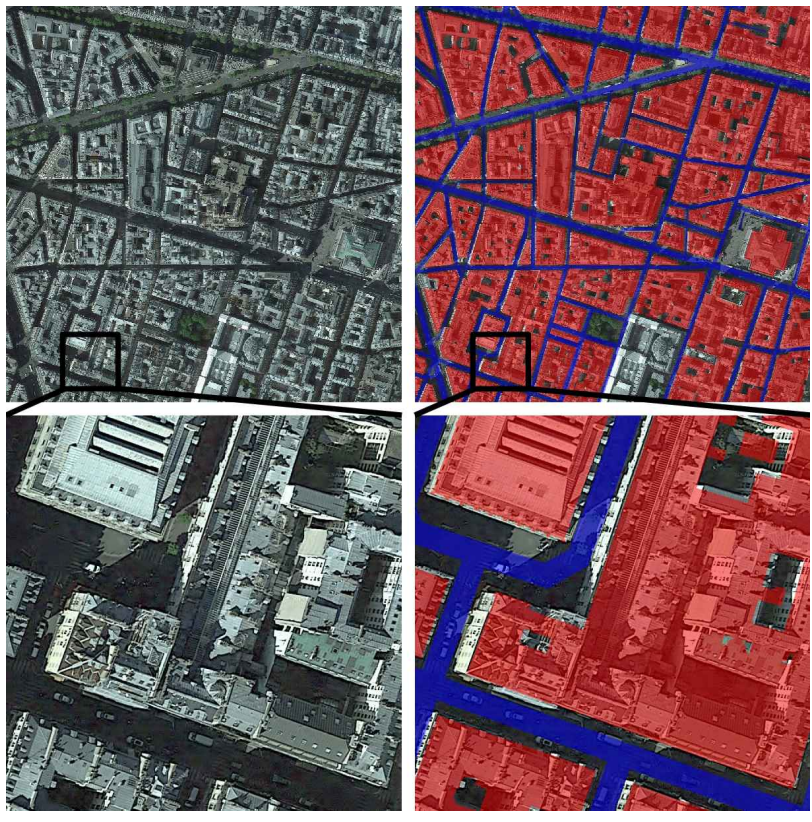

(b)

Fig. 1. Example of OSM labels overlaid with Google Maps images for (a) Zurich and (b) Paris. The left side shows an aerial image and a magnified detail. The right side shows the same images overlaid with building (red) and road (blue) labels. Background is transparent in the label map.

inside a $(2 \times 2)$ neighborhood. The downsampling increases the receptive field of subsequent convolutions, and lets the network learn correlations over a larger spatial context. Moreover, maxpooling achieves local translation invariance at object level. The outputs of the last convolutional layers (which are very big to capture global context, equivalent to a fully connected layer of standard CNNs) is converted to a vector of scores for the three target classes. These score maps are of low resolution, hence they are gradually upsampled again with convolutional layers using a stride of only $\frac{1}{2}$ pixel $]^{7}$ Repeated downsampling causes a loss of high-frequency content, which leads to blurry boundaries that are undesirable for pixel-wise semantic segmentation. To counter this effect, feature maps at intermediate layers are merged back in during upsampling (socalled "skip connections", see Fig. 22. The final, full-resolution score maps are then converted to label probabilities with the softmax function.

\section{Implementation Details}

The FCN we use is an adaptation of the architecture proposed in [5], which itself is largely based on the VGG-16 network architecture [41]. In our implementation, we slightly modify the original FCN and introduce a third skip connection (marked red in Fig. 2), to preserve even finer image details. We found that the original architecture, which has two skipconnections after Pool_3 and Pool_4 (cf. Fig. 3) was still not delivering sufficiently sharp edges. The additional, higherresolution skip connection consistently improved the results

\footnotetext{
${ }^{7}$ This operation is done by layers that are usually called "deconvolution layers" in the literature [5] (and also in Fig. 3] although the use of this terminology has been critized since most implementations do not perform a real deconvolution but rather a transposed convolution.
}

for our data, see Sec. IV-B Note that adding the third skipconnection does not increase the total number of parameters but, on the contrary, slightly reduces it ([5]: $134^{\prime} 277^{\prime} 737$, ours: $134^{\prime} 276^{\prime} 540$; the small difference is due to the decomposition of the final upsampling kernel into two smaller ones).

\section{Training}

All model parameters are learned by minimising a multinomial logistic loss, summed over the entire $500 \times 500$ pixel patch that serves as input to the FCN. Prior to training/inference, intensity distributions are centred independently per patch by subtracting the mean, separately for each channel (RGB).

All models are trained with stochastic gradient descent with a momentum of 0.9 , and minibatch size of 1 image. Learning rates always start from $5 \times 10^{-9}$ and are reduced by a factor of 10 twice when the loss and average $F_{1}$ scores stopped improving. The learning rates for biases of convolutional layers were doubled with respect to learning rates of the filter weights. Weight decay was set to $5 \times 10^{-4}$, dropout probability for neurons in layers ReLU_6 and ReLU_7 was always 0.5 .

Training was run until the average $F_{1}$-score on the validation dataset stopped improving, which took between 45000 and 140000 iterations (3.5-6.5 epochs). Weights were initialized as in Glorot et al. [52], except for experiments with pre-trained weights. It is a common practice in deep learning to publish pre-trained models together with source code and paper, to ease repeatability of results and to help others avoid training from scratch. Starting from pre-trained models, even if these have been trained on a completely different image dataset, often improves performance, because low-level features like contrast edges and blobs learned in early network layers are very similar across different kinds of images. 


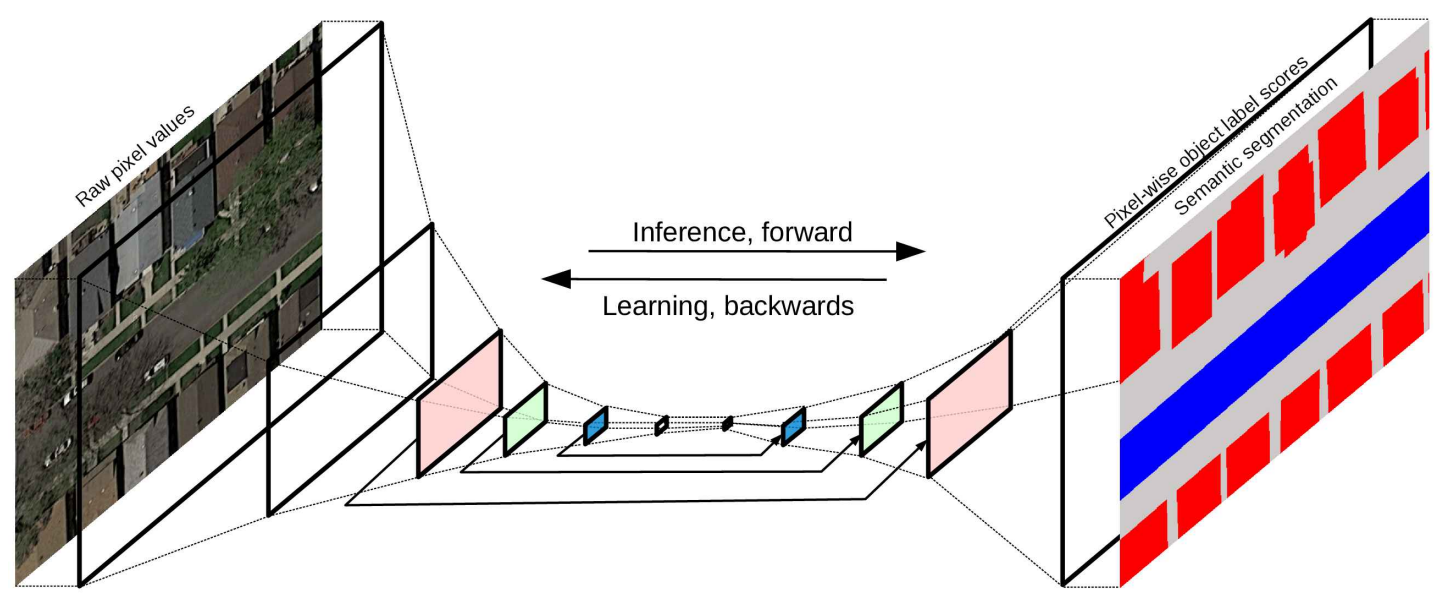

Fig. 2. Conceptual illustration of the data flow through our variant of a fully convolutional network (FCN), which is used for the semantic segmentation of aerial images. Three skip-connections are highlighted by pale red, pale green, and pale blue, respectively. Note that we added a third (pale red) skip connection in addition to the original ones (pale green, pale blue) of [5].

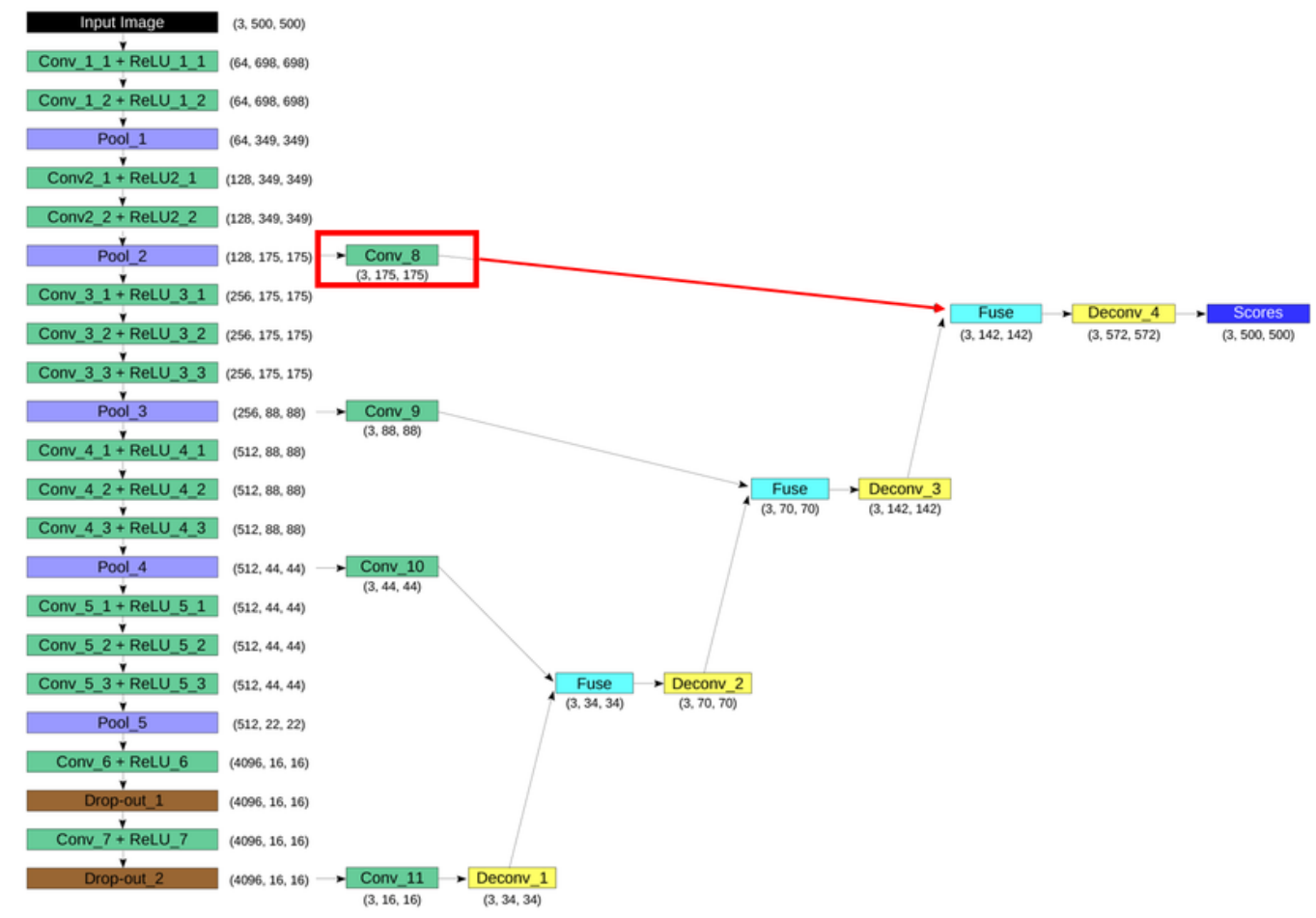

Fig. 3. Our FCN architecture, which adds one more skip-connection (after Pool_2, shown red) to the original model of [5]. Neurons form a three-dimensional structure per layer: dimensions are written in brackets, where the first number indicates the amount of feature channels, second and third represent spatial dimensions.

We will use two different forms of pre-training. Either we rely on weights previously learned on the Pascal VOC benchmark [53] (made available by the authors of [5]). Or we pre-train ourselves with OSM data. In the experiments section it is always specified whether we use VOC, OSM or no pretraining at all.

\section{EXPERIMENTS}

We present extensive experiments on four large data sets of different cities to explore the following scenarios:

- Complete substitution: Can semantic segmentation be learned without any manual labeling? What performance can be achieved using only noisy labels gleaned from OSM?

- Augmentation: Will pre-training with large-scale OSM data and publicly available images improve the segmentation of a project-specific data set of independently acquired images and labels?

- Partial substitution: Can pre-training with large-scale OSM labels replace a substantial part of the manual labeling effort? Phrased differently, can a generic model learned from OSM be adapted to a specific location and data source with only little dedicated training data? 
We provide a summary of the results and explicit answers to these questions at the very end of this section. Note that all experiments are designed to investigate different aspects of the hypotheses made in the introduction. We briefly remind and thoroughly validate all hypotheses based on results of our experiments in the conclusion.

\section{A. Datasets}

Four large datasets were downloaded from Google Maps and OSM, for the cities of Chicago, Paris, Zurich, and Berlin. Additionally we also downloaded a somewhat smaller dataset for the city of Potsdam. For this location, a separate image set and high-accuracy ground truth is available from the ISPRS semantic labeling benchmark [54]. Table I] specifies the coverage (surface area), number of pixels, and ground sampling distance of each dataset. Example images and segmentation maps of Paris and Zurich are shown in Figure 1. In Fig 4 we show the full extent of the Potsdam scene, dictated by the available images and ground truth in the ISPRS benchmark. OSM maps and aerial images from Google Maps where downloaded and cut to cover exactly the same region to ensure a meaningful comparison - this meant, however, that the dataset is an order of magnitude smaller than what we call "large-scale" for the other cities. The ISPRS dataset includes a portion (images $x_{-} 13, x_{-} 14, x_{-} 15$ on the right side of Fig. 4), for which the ground truth is withheld to serve as test set for benchmark submissions. We thus use images 2_12,6_10, and 7_11 as test set, and the remaining ones for training. The three test images were selected to cover different levels of urban density and architectural layout. This train-test split corresponds to $1.89 \mathrm{~km}^{2}$ of training data, respectively $0.27 \mathrm{~km}^{2}$ of test data.

The ISPRS semantic labeling challenge aims at land-cover classification, whereas OSM represents land-use. In particular, the benchmark ground truth does not have a label street, but instead uses a broader class impervious surfaces, also comprising sidewalks, tarmacked courtyards etc. Furthermore, it labels overhanging tree canopies that occlude parts of the impervious ground (including streets) as tree, whereas streets in the OSM labels include pixels under trees. Moreover, images in the ISPRS benchmark are "true" orthophotos rectified with a DSM that includes buildings, whereas Google images are conventional orthophotos. corrected only for terrain-induced distortions with a DTM. Building facades remain visible and roofs are shifted from the true footprint. To facilitate a meaningful comparison, we have manually re-labeled the ISPRS ground truth to our target categories street, and background, matching the land-use definitions extracted from OSM. The category building of the benchmark ground truth remains unchanged. To allow for a direct and fair comparison, we down-sample the ISPRS Potsdam data, which comes at a ground sampling distance (GSD) of $5 \mathrm{~cm}$, to the same GSD as the Potsdam-Google data $(9.1 \mathrm{~cm})$.

For all datasets, we cut the aerial images as well as the corresponding label maps into non-overlapping tiles of size $500 \times 500$ pixels. The size was determined in preliminary experiments, to include sufficient geographical context while keeping FCN training and prediction efficient on a normal

\begin{tabular}{|l|c|c|c|}
\hline & Coverage & No. of pixels & GSD \\
\hline Chicago & $50.6 \mathrm{~km}^{2}$ & $4.1 \times 10^{9}$ & $11.1 \mathrm{~cm}$ \\
Paris & $60.3 \mathrm{~km}^{2}$ & $6.3 \times 10^{9}$ & $9.8 \mathrm{~cm}$ \\
Zurich & $36.2 \mathrm{~km}^{2}$ & $3.5 \times 10^{9}$ & $10.1 \mathrm{~cm}$ \\
Berlin & $10.6 \mathrm{~km}^{2}$ & $1.28 \times 10^{9}$ & $9.1 \mathrm{~cm}$ \\
\hline Potsdam-Google & $2.16 \mathrm{~km}^{2}$ & $2.60 \times 10^{8}$ & $9.1 \mathrm{~cm}$ \\
Potsdam-ISPRS & $2.16 \mathrm{~km}^{2}$ & $2.60 \times 10^{8}$ & $9.1 \mathrm{~cm}$ \\
\hline
\end{tabular}

TABLE I

STATISTICS OF THE DATASETS USED IN THE EXPERIMENTS. NOTE THAT WE DOWN-SAMPLED THE ORIGINAL POTSDAM-ISPRS $(G S D=5 \mathrm{~cm})$ TO THE RESOLUTION OF THE POTSDAM-GOOGLE DATA $(G S D=9.1 \mathrm{~cm})$ FOR ALL EXPERIMENTS.

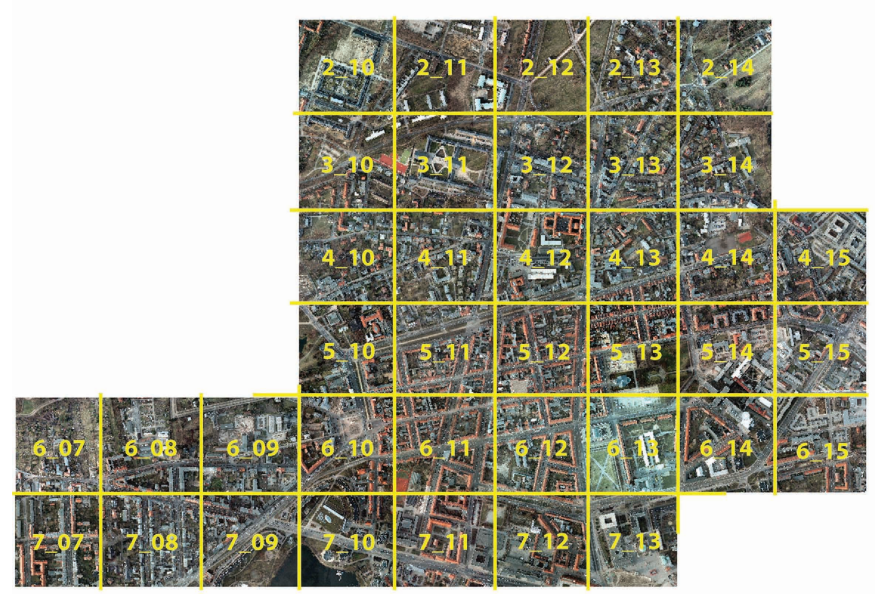

Fig. 4. Overview of the ISPRS Potsdam dataset. The aerial images shown are those provided by the ISPRS benchmark [54].

single-GPU desktop machine. Each dataset is split into mutually exclusive training, validation and test regions. During training, we monitor the loss (objective function) not only on the training set, but also on the validation set to prevent overfitting 8

\section{B. Results and discussion}

First, we validate our modifications of the FCN architecture, by comparing it to the original model of [5]. As error metrics, we always compute precision, recall and $F_{1}$-score, per class as well as averaged over all three classes. Precision is defined as the fraction of predicted labels that are correct with respect to ground truth, recall is the fraction of true labels that are correctly predicted. The $F_{1}$-score is the harmonic mean between precision and recall. It combines the two competing goals into a scalar metric and is widely used to assess semantic segmentation. It also serves as our primary error measure. Quantitative results are shown in Table [I] an example result for Chicago is shown in Figure 5. Our architecture with the additional early skip-connection outperforms its counterpart slightly but consistently on average, albeit only by $\approx 1$ percent point. Note that this performance improvement also

\footnotetext{
${ }^{8}$ This is standard practice when training deep neural networks.
} 


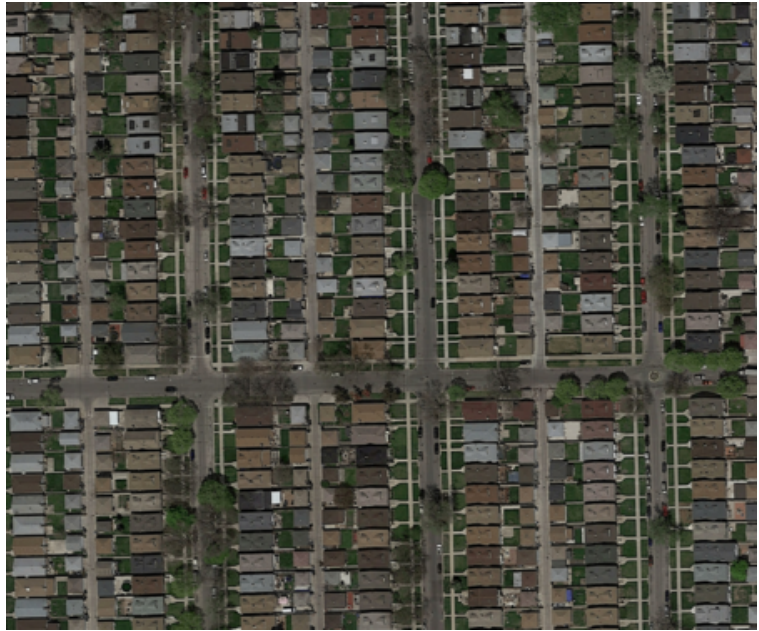

(a)

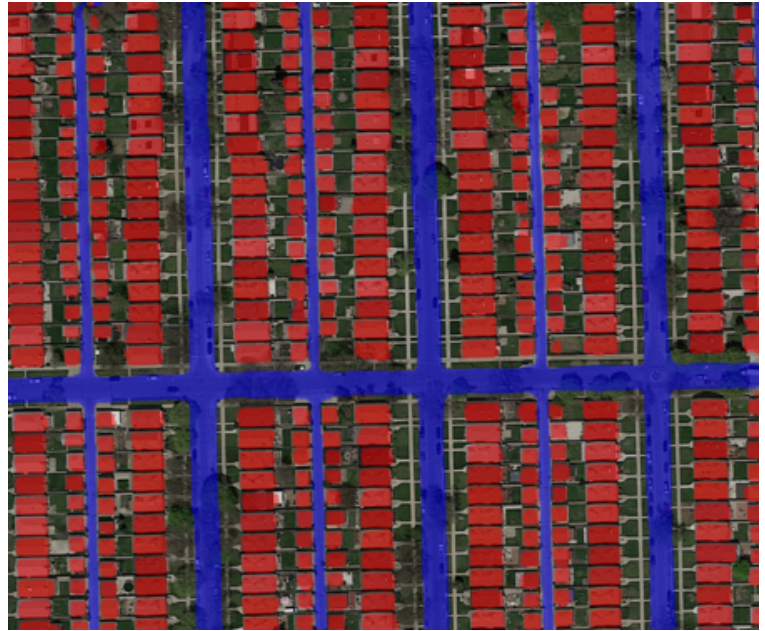

(b)

Fig. 5. FCN trained on Google Maps imagery and OSM labels of Chicago: (a) Original aerial image and (b) overlaid with classification result.

comes with the benefit of lower run-times. Our architecture consistently needs $\geq 30 \%$ less time for training compared to the original architecture of [5] (see Table II).

Another interesting finding is in terms of transfer learning, in the sense that training a model over multiple cities, with both different global scene structure and different object appearance, can help better predict a new, previously unseen city. This again emphasizes the improved generalization ability that benefits from the increased amount of weak labels, in contrast to traditional supervised approaches with smaller label sets. We train the FCN on Zurich, Paris, and Chicago and predict Tokyo. We compare the results with those from training on only a single city (Fig 6). It turns out that training over multiple, different cities helps the model to find a more general, "mean" representation of what a city looks like. Generalising from a single city to Tokyo clearly performs worse (Fig. 6(a,b,c)) than generalising from several different ones (Fig. 6(d)). This indicates that FCNs are indeed able to learn location-specific urbanistic and architectural patterns; but also that supervision with a sufficiently diverse training set mitigates this effect and still lets the system learn more global, generic patterns that support semantic segmentation in different geographic regions not seen at all during training.

For experiments on the ISPRS Potsdam data set, we first compute three baselines. For an overview of the setup of all experiments described in the following, please refer to Table III] whereas quantitative results are given in Table IV.

(I) Baseline with ISPRS data: First, we follow the conventional semantic segmentation baseline and apply our FCN model to the ISPRS benchmark to establish a baseline with conventional, hand-labeled ground truth. As a training set of realistic size we use three completely labelled images from the ISPRS Potsdam benchmark $\left(3.25 \cdot 10^{7}\right.$ pixels / $\left.27 \mathrm{ha}\right)$. This setup Ia achieves 0.764 average $F_{1}$-score over the three classes if we train our FCN from scratch, i.e., weights initialized randomly as in [52] (Fig. 7(a,b,c)). A widely used practice is to start from a pre-trained model that has been learned from a very large dataset, especially if the dedicated training data is limited in size. We thus compute baseline Ib, where we start from a model trained on the Pascal VOC benchmark and finetune on the three ISPRS Potsdam images. As expected this boosts performance, to 0.809 average $F_{1}$-score (Fig. $7(\mathrm{~d}, \mathrm{e}, \mathrm{f})$ ).

(II) Gold standard with ISPRS data: Second, we repeat the same experiment, but use all of the available training data, i.e., we train on all 21 available training images $\left(2.275 \cdot 10^{8}\right.$ pixels / 189 ha). This setup serves as a "gold standard" for what is achievable with the conventional pipeline, given an unusually large amount of costly high-quality training labels. It simulates a project with the luxury of $>200$ million handlabelled training pixels over a medium-sized city (which will rarely be the case in practice). It achieves an $F_{1}$-score of 0.874 if trained from scratch (Fig. 77). The significant improvement of 11 , respectively 6 percent points shows that our "standard" baselines Ia and Ib are still data-limited, and can potentially be improved significantly with additional training data. As a sanity check, we also ran the same experiment with all 21 ISPRS images and pre-training from Pascal VOC. This only marginally increases the average $F_{1}$-score to 0.879 .

We note that baseline II is not directly comparable with the existing benchmark entries, since we work with a reduced class nomenclature and modified ground truth, and do not evaluate on the undisclosed test set. But it lies in a plausible range, on par with or slightly below the impervious ground and building results of the competitors, who, unlike us, also use the DSM.

(III) Baseline with Google Maps images and OSM Maps: The next baseline IIIa trains on Google aerial images using OSM map data as ground truth. The same 189 ha as in baseline II are used for training, and the model achieves an $F_{1}$-score of 0.777 if tested on Google aerial images and OSM ground truth (Fig. $8(a, b, c))$. This baseline has been added as a sanity check to verify that the previously observed potential of the open data sources is confirmed also for Potsdam. We point out that the experiment is somewhat problematic and not comparable to baseline II, in that it inevitably confounds several effects: the drop in performance may in part be due to the larger amount of noise in the training labels; but further possible reasons 


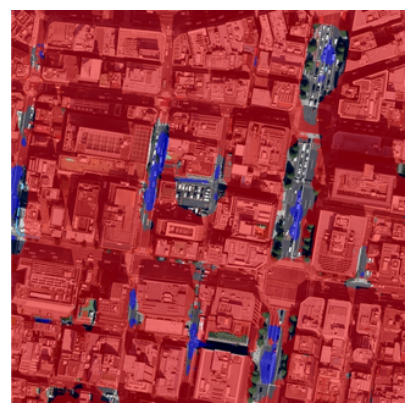

(a)

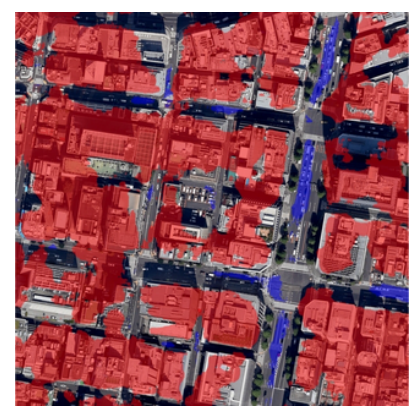

(b)

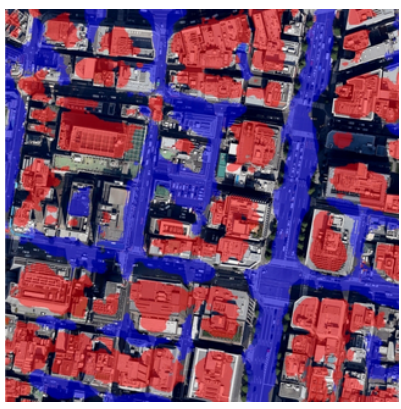

(c)

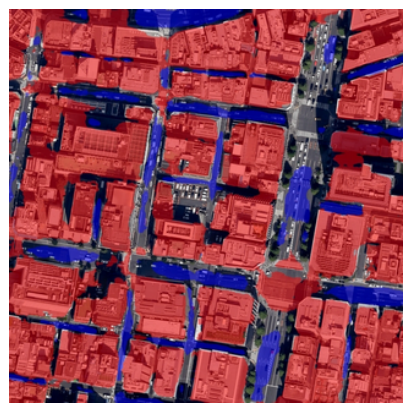

(d)

Fig. 6. Classification results and average $F^{1}$-scores of the Tokyo scene with a model trained on (a) Chicago $\left(F^{1}: 0.485\right)$, (b) Paris $\left(F^{1}: 0.521\right)$, (c) Zurich $\left(F^{1}: 0.581\right),(d)$ all three $\left(F^{1}: 0.644\right)$.

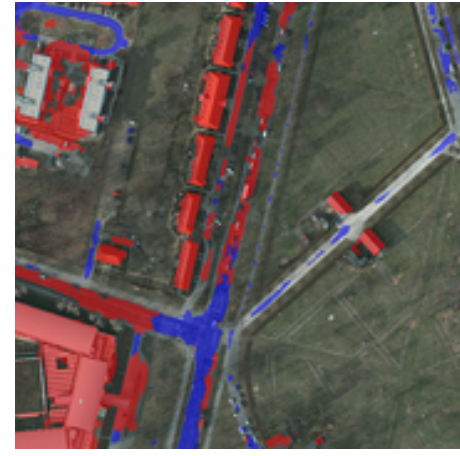

(a)

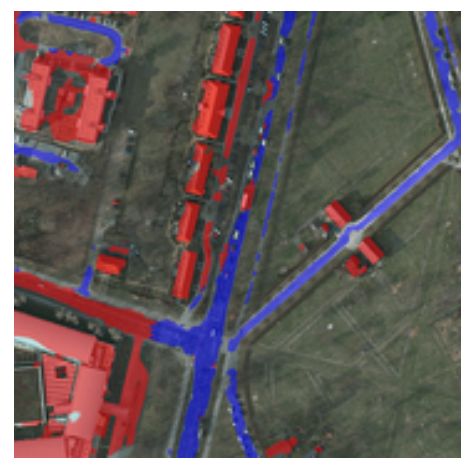

(d)

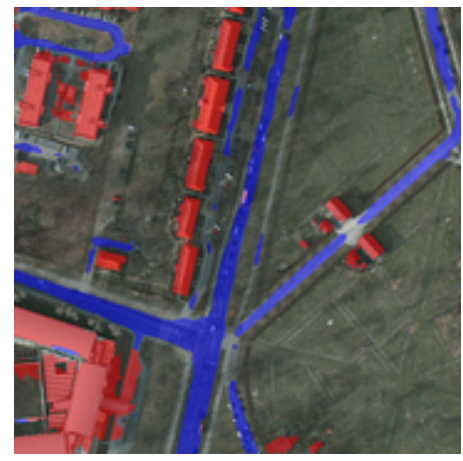

(g)

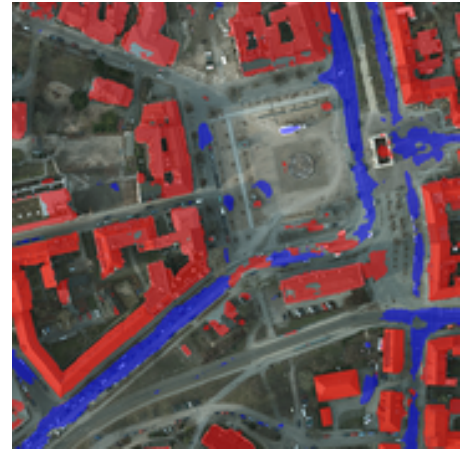

(b)

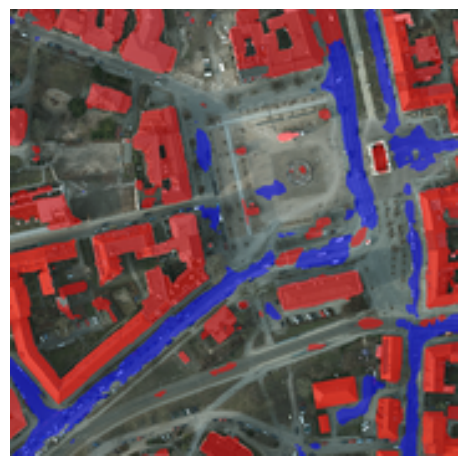

(e)

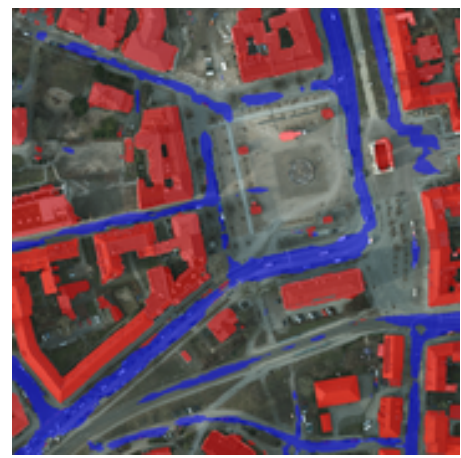

(h)

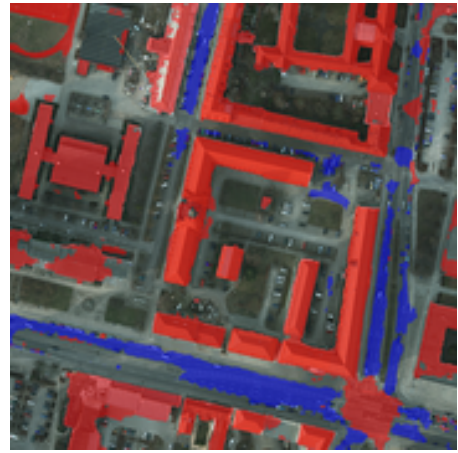

(c)

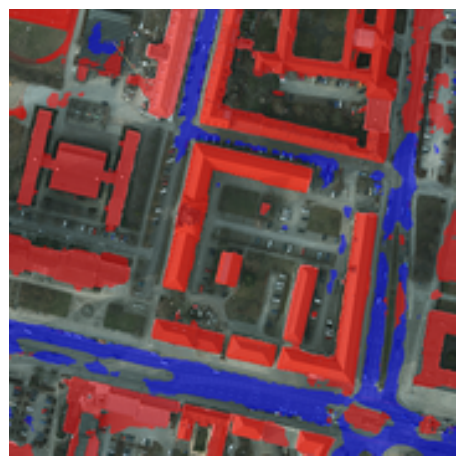

(f)

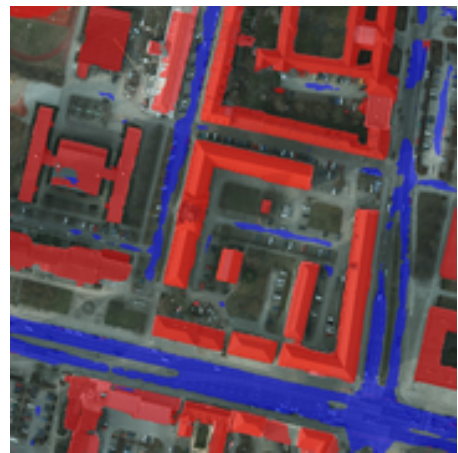

(i)

Fig. 7. Baseline experiments, (a,b,c): Baseline Ia trained on three ISPRS images without pre-training. (d,e,f): Baseline Ib trained on three ISPRS images with pre-training on Pascal VOC. (g,h,i): Gold standard II trained on 21 ISPRS images. 


\begin{tabular}{|l|c|c|c|c|c|c|}
\hline & \multicolumn{2}{|c|}{ Chicago } & \multicolumn{2}{c|}{ Paris } & \multicolumn{2}{c|}{ Zurich } \\
\hline & {$[5](15.7 \mathrm{~h})$} & Ours $(10.5 \mathrm{~h})$ & {$[5](18.3 \mathrm{~h})$} & Ours $(7.6 \mathrm{~h})$ & {$[5](15.5 \mathrm{~h})$} & Ours $(6.2 \mathrm{~h})$ \\
\hline$F_{1}$ average & 0.840 & $\mathbf{0 . 8 5 5}$ & 0.774 & $\mathbf{0 . 7 7 6}$ & 0.804 & $\mathbf{0 . 8 1 0}$ \\
\hline$F_{1}$ building & 0.823 & $\mathbf{0 . 8 3 7}$ & 0.821 & $\mathbf{0 . 8 2 2}$ & $\mathbf{0 . 8 2 4}$ & 0.823 \\
\hline$F_{1}$ road & 0.821 & $\mathbf{0 . 8 4 3}$ & 0.741 & $\mathbf{0 . 7 4 6}$ & 0.695 & 0.707 \\
\hline$F_{1}$ background & 0.849 & $\mathbf{0 . 8 6 1}$ & $\mathbf{0 . 7 5 4}$ & $\mathbf{0 . 7 5 4}$ & $\mathbf{0 . 8 9 4}$ & 0.891 \\
\hline
\end{tabular}

TABLE II

COMPARISON BETWEEN OUR ADAPTED FCN AND THE ORIGINAL ARCHITECTURE OF [5], FOR THREE LARGE CITY DATASETS. NUMBERS IN BRACKETS INDICATE TRAINING TIMES FOR THE ORIGINAL FCN ARCHITECTURE OF [5] AND OURS FOR ALL DATA SETS IF TRAINED FROM SCRATCH WITHOUT ANY PRE-TRAINING TO FACILITATE A FAIR COMPARISON (ON A STANDARD, STAND-ALONE PC WITH I7 CPU, 2.7 GHZ, 64 GB RAM AND NVIDIA TITAN-X GPU WITH 12 GB RAM).

\begin{tabular}{|c|c|c|c|c|c|c|}
\hline & & \# ISPRS images & pre-training & OSM & Google images & test data \\
\hline Ia & ISPRS baseline & 3 & no & - & - & ISPRS \\
\hline Ib & ISPRS baseline pre-trained & 3 & yes & - & - & ISPRS \\
\hline II & ISPRS gold standard & 21 & yes \& no & - & - & ISPRS \\
\hline IIIa & Google/OSM baseline Potsdam & - & no & $\mathrm{P}$ & $\mathrm{P}$ & OSM+Google \\
\hline IIIb & Google/OSM baseline Potsdam+Berlin & - & yes & $\mathrm{P}, \mathrm{B}$ & $\mathrm{P}, \mathrm{B}$ & OSM+Google \\
\hline IV & Complete substitution & 21 & no & $P$ & - & ISPRS \\
\hline $\mathbf{V}$ & Augmentation & 21 & yes & $\mathrm{B}, \mathrm{Z}, \mathrm{C}, \mathrm{P}$ & $\mathrm{B}, \mathrm{Z}, \mathrm{C}, \mathrm{P}$ & ISPRS \\
\hline VI & Partial substitution & 3 & yes & $\mathrm{B}, \mathrm{Z}, \mathrm{C}, \mathrm{P}$ & $\mathrm{B}, \mathrm{Z}, \mathrm{C}, \mathrm{P}$ & ISPRS \\
\hline
\end{tabular}

TABLE III

OVERVIEW OF DIFFERENT EXPERIMENTAL SETUPS WE USE TO VALIDATE OUR HYPOTHESIS MADE IN THE INTRODUCTION. WE ABBREVIATE BERLIN (B), Zurich (Z), ChicAGo (C), AND POTSDAM (P). All ENTRIES REFER TO THE TRAining SETUP EXCEPT THE MOST RIGHT COLUMN, Which INDICATES DATA USED FOR TESTING. QUANTITATIVE RESULTS FOR ALL EXPERIMENTS ARE GIVEN IN TABLEIV

include on the one hand the inferior image quality of the Google Maps images, c.f. cast shadows and ortho-rectification artifacts in Fig. 8(b,c); and on the other hand the noise in the OSM-based test labels 9 Recall that the same setup achieved 0.810 for the architecturally comparable Zurich, and 0.827 for the more schematic layout of Chicago. This suggests that a part of the drop may be attributed to the smaller training set, respectively that noisy OSM labels should be used in large quantities. To verify this assumption we repeat the experiment, but greatly extend the training dataset by adding the larger city of Berlin, which is immediately adjacent to Potsdam. This baseline IIIb increases performance by 2 percent points to 0.797 (Fig. 8. d,e,f)), which is only slightly below performance on Zurich (0.810). It shows that training data size is a crucial factor, and that indeed city-scale (though noisy) training data helps to learn better models.

Qualitatively, one can see that the model trained on OSM has a tendency to miss bits of the road, and produces slightly less accurate and blurrier building outlines.

(IV) Complete substitution of manual labels: Next, we evaluate the extreme setting where we do not have any highaccuracy labels and completely rely on OSM as source of

\footnotetext{
${ }^{9}$ We also test the same model on Google aerial images with ISPRS labels, which leads to a slight performance drop to 0.759. This is not surprising, because labels have been acquired based on the ISPRS images and do not fit as accurately to the Google images.
}

training data. We thus train our FCN on the ISPRS Potsdam images, but use OSM map data as ground truth. The predictions for the ISPRS test images are then evaluated with the manual high-accuracy ground truth from the benchmark. In other words, this experiments quantifies how accurate predictions we can expect if training from OSM labels for a limited, project-specific image set: since the ISPRS dataset does not provide more images, one cannot augment the training set further, even though a lot more OSM data would be available. This set up achieves an $F_{1}$-score of 0.779 , beating baseline Ia by 1.5 percent points. We conclude that larger amounts of noisy, automatically gleaned training data can indeed completely replace small amounts of highly accurate training data, saving the associated effort and cost. The result however does stay 3 percent points behind baseline $\mathbf{I b}$, which shows that even all of Potsdam is not large enough to replace pre-training with large-scale data, which will be addressed in experiment VI. Compared to baseline II, i.e. training with equally large quantities of pixel-accurate labels, performance drops by 10 percent points. The visual comparison between baseline II in Fig. 7 ( $\mathrm{g}, \mathrm{h}, \mathrm{i})$ and IV in Fig. 9(a,b,c) shows that buildings are segmented equally well, but roads deteriorate significantly. This is confirmed by the $F_{1}$-scores in Table IV] An explanation is the noise in the guessed road width (as also pointed out by [55]) in the training data ( $\approx 23$ pixels on average, for an 


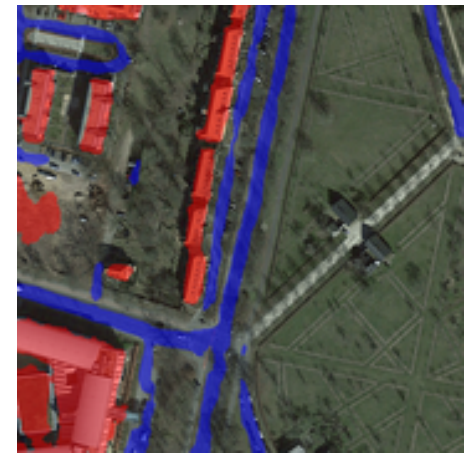

(a)

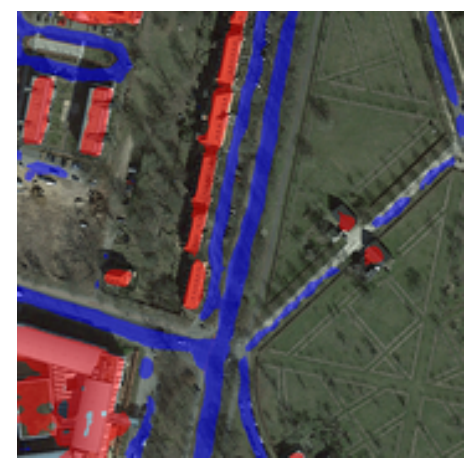

(d)

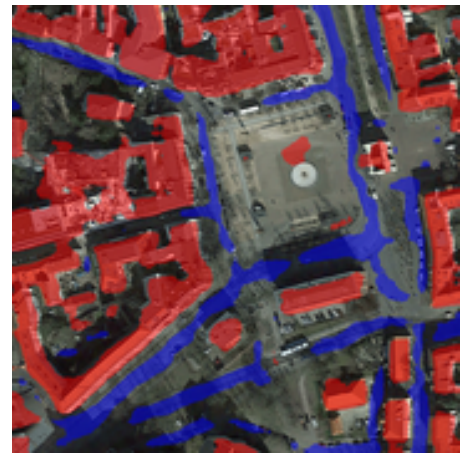

(b)

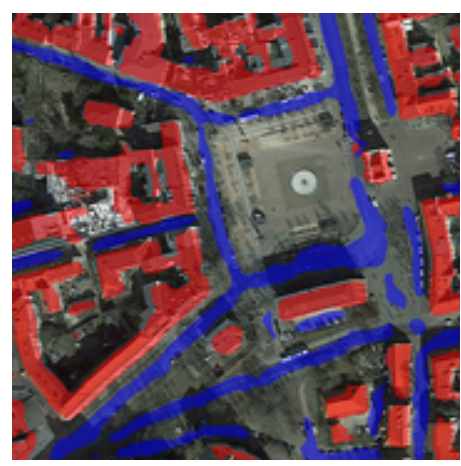

(e)

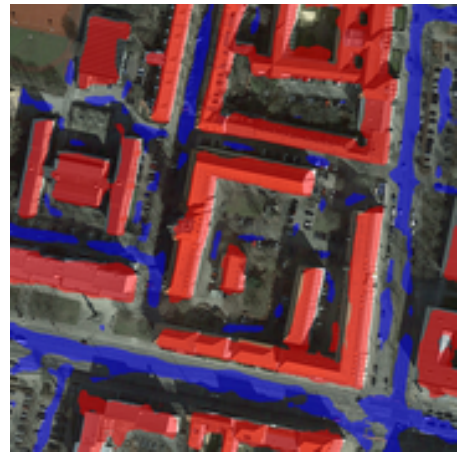

(c)

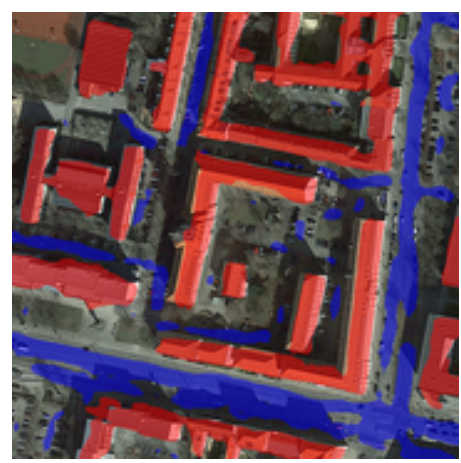

(f)

Fig. 8. Baseline experiments, (a,b,c): Baseline IIIa with Google Maps images and OSM Maps from only Potsdam. (d,e,f): Baseline IIIb with Google Maps images and OSM Maps and training on Potsdam and Berlin.

average road width of $\approx 100$ pixels). It leads to washed-out evidence near the road boundaries, which in turn weakens the overall evidence in the case of narrow or weakly supported roads. This effect can be observed visually by comparing probability maps of II and IV in Fig. 10 Road probabilities appear much sharper at road edges for baseline II trained with pixel-accurate ISPRS groundtruth (Fig. 10(a,b,c)) compared to IV trained with noisy OSM ground truth (Fig. 10(d,e,f)).

(V) Augmentation with open data: With experiment $\mathbf{V}$ we aim to assess whether pre-training from even larger amounts of open data from other sites can further improve the goldstandard II, by providing a sort of "generic background" for the problem, in the spirit of pre-trained computer vision models such as VGG [41] or Alexnet [40]. We first train the FCN model on Google/OSM data of Chicago, Paris, Zurich, and Berlin, and use the resulting network weights as initial value, from which the model is tuned for the ISPRS data, using all the 21 training images as in baseline II. The pre-training boosts performance, albeit only by 1 percent point. Even if one has a comfortable amount of accurate training data at hand, it appears potentially useful to pre-train with freely available data. In future work it may be useful to experiment with even larger amounts of open data.

A visual comparison of Fig. 7 ( $\mathrm{g}, \mathrm{h}, \mathrm{i})$ and Fig. 9 (d,e,f) shows small improvements for both the roads and the buildings, in all three tiles. This effect shows up quantitatively with an improvement in $F_{1}$-score of the road class, which reaches 0.825 , up from 0.764 in baseline II. On the other hand, buildings are detected equally well, no further improvement can be noticed. A possible interpretation is that complex network structures with long-range dependencies are hard to learn for the classifier, and thus more training data helps. Locally well-defined, compact objects of similar shape and appearance are easier to learn, so further training data does not add relevant information.

(VI) Partial substitution of manual labels: The success of pre-training in previous experiments raises the question - also asked in [50] - of whether one could reduce the annotation effort and use a smaller hand-labelled training set, in conjunction with large-scale OSM labels. An alternative view is as a domain adaptation problem, where the classifier is trained on Google Maps images, and then re-targeted to ISPRS images with only few training samples. The hope is that the large amount of OSM training data would already allow the classifier to learn basic aerial image statistics and urban scene structures. Then, only a small additional training set would suffice to adapt it to the different spectral properties. In experiment VI we therefore first train the FCN on the combined Google / OSM data of Chicago, Paris, Zurich, and Berlin. This part is the same as in experiment $\mathbf{V}$. Then, we use only the small set of training images and labels from baseline I to tune it to the ISPRS images of Potsdam. Performance increases by 7 percent points to 0.837 over baseline Ia, where the model is trained from scratch on the same high-accuracy labels. We conclude that if only a limited quantity of highquality training data is available, pre-training on free data 


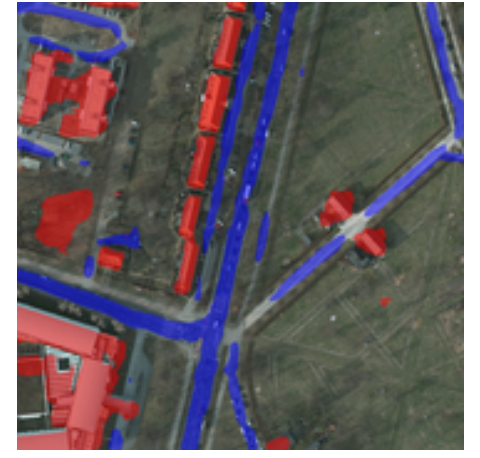

(a)

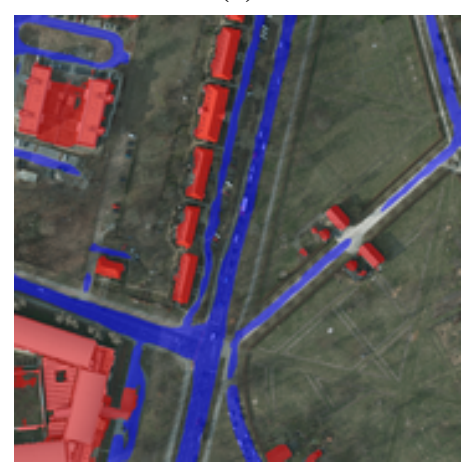

(d)

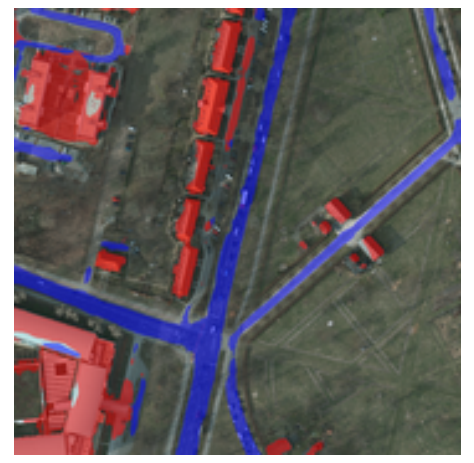

(g)

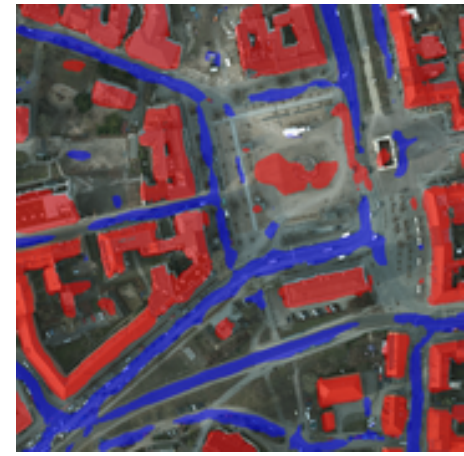

(b)

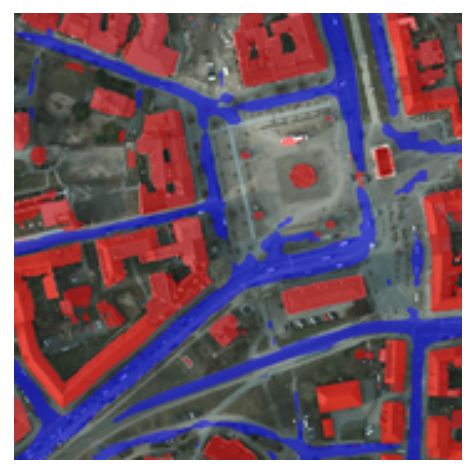

(e)

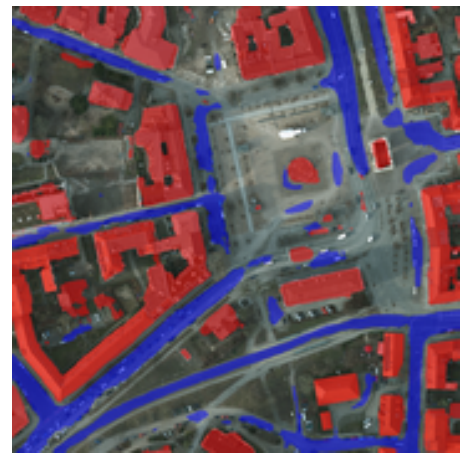

(h)

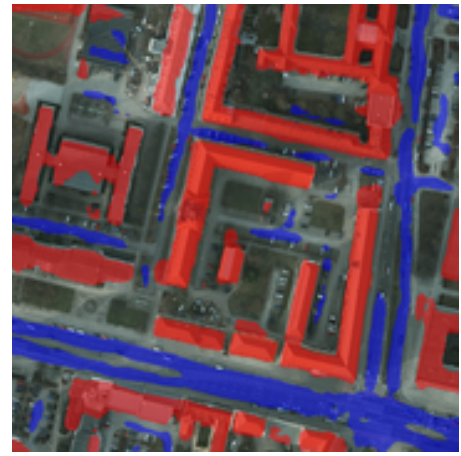

(c)

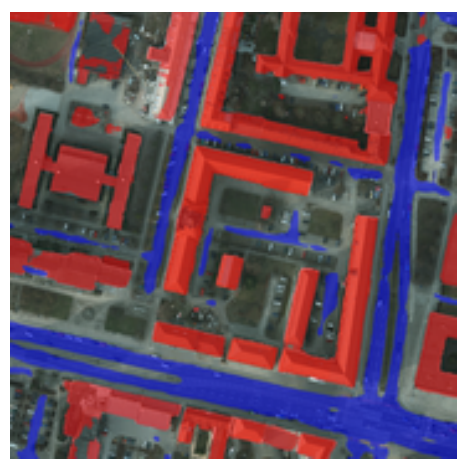

(f)

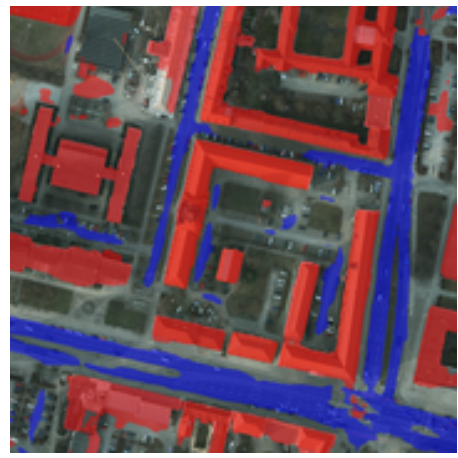

(i)

Fig. 9. (a,b,c): Complete substitution (IV) of manual labels, train from scratch on ISPRS images and OSM labels of Potsdam (no pre-training). (d,e,f): Augmentation $(\mathbf{V})$ with open data, pre-train on Chicago, Paris, Zurich, and Berlin and re-train on all 21 ISPRS training images with pixel-accurate ground truth. (g,h,i): Partial substitution (VI) of manual labels, pre-train on Chicago, Paris, Zurich, and Berlin and re-train on 3 ISPRS images with pixel-accurate ground truth.

brings even larger relative benefits, and can be recommended as general practice, which is in line with the findings reported in [50].

Importantly, experiment VI also outperforms baseline Ib by almost 3 percent points, i.e., pre-training on open geo-spatial and map data is more effective than using a generic model pre-trained on random web images from Pascal VOC. While pre-training is nowadays a standard practice, we go one step further and pre-train with aerial images and the correct set of output labels, generated automatically from free map data.

Compared to the gold standard baseline II the performance is $\approx 4$ percent points lower ( 0.837 vs. 0.874$)$. In other words, fine-tuning with a limited quantity of problem-specific highaccuracy labels compensates a large portion $(\approx 65 \%)$ of the loss between experiments II and IV, with only $15 \%$ of the labeling effort. Relative to II, buildings degrade most ( 0.863 vs. 0.913). This can possibly be attributed to the different appearance of buildings due to different ortho-rectification. Recall that Google images were rectified with a DTM and are thus geometrically distorted, with partially visible facades. It seems that fine-tuning with only three true orthophotos $(<100$ buildings) is not sufficient to fully adjust the model to the different projection.

Pushing the "open training data" philosophy to the extreme, one could ask whether project-specific training is necessary at all. Maybe the model learned from open data generalizes even to radiometrically different images of comparable GSD? We do not expect this to work, but as a sanity check for a "generic, 


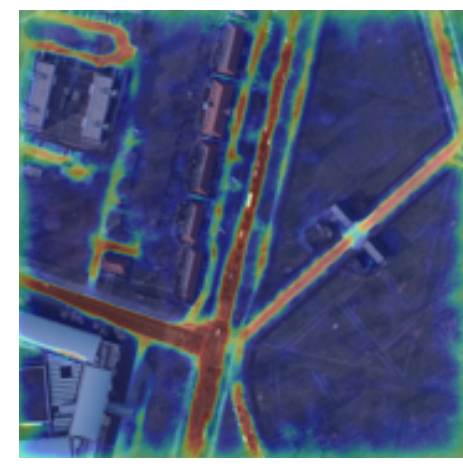

(a)

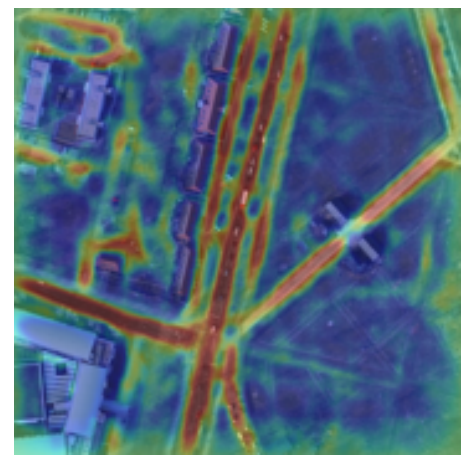

(d)

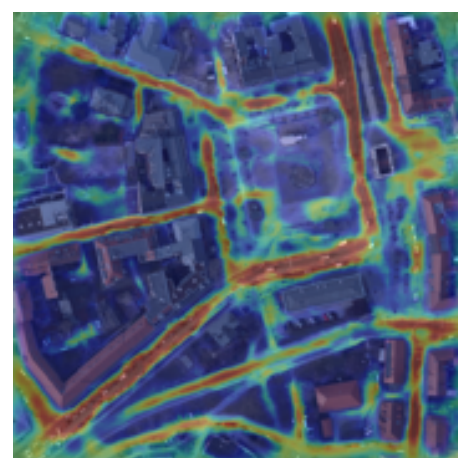

(b)

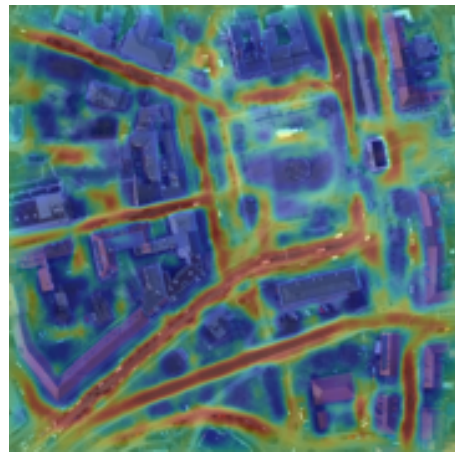

(e)

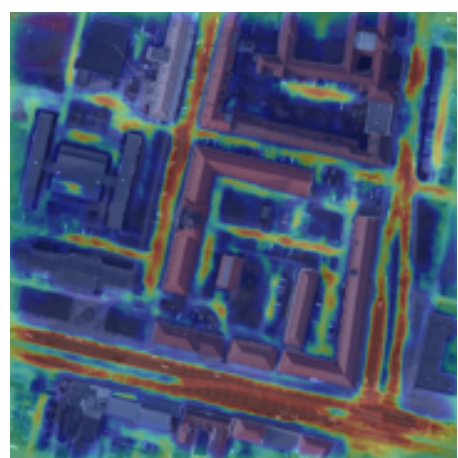

(c)

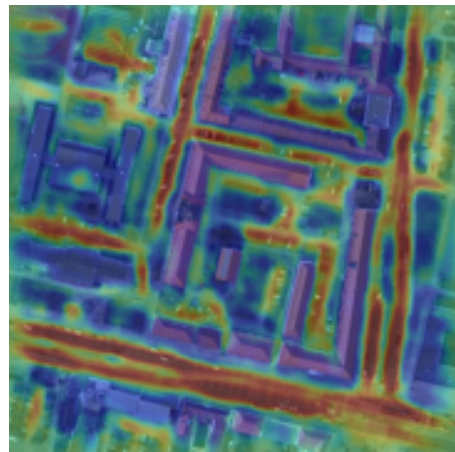

(f)

Fig. 10. Probability maps for road extraction of the gold standard baseline II (a,b,c); and complete substitution IV without any manual labels (d,e,f). Road probabilities range from red (high) to blue (low).

\begin{tabular}{|l|l|c|c|c||c|c|c|c|}
\cline { 3 - 9 } \multicolumn{2}{l|}{} & av. $F_{1}$ & av. Precision & av. Recall & $F_{1}$ Building & $F_{1}$ Road & $F_{1}$ Background & train time $[\mathrm{h}]$ \\
\hline Ia & ISPRS baseline & 0.764 & 0.835 & 0.704 & 0.793 & 0.499 & 0.883 & 16 \\
\hline Ib & ISPRS baseline pre-trained & 0.809 & 0.853 & 0.770 & 0.830 & 0.636 & 0.904 & 16 \\
\hline II & ISPRS gold standard & $\underline{0.874}$ & $\mathbf{0 . 9 1 0}$ & $\underline{0.841}$ & $\mathbf{0 . 9 1 3}$ & $\underline{0.764}$ & $\mathbf{0 . 9 2 3}$ & 16 \\
\hline IIIb & Google/OSM baseline P & 0.777 & 0.799 & 0.756 & 0.832 & 0.631 & 0.845 & 16 \\
\hline \hline IV & Google/OSM baseline P+B & 0.797 & 0.819 & 0.776 & 0.828 & 0.698 & 0.858 & 32 \\
\hline V & Augmentation & 0.779 & 0.801 & 0.758 & 0.796 & 0.667 & 0.860 & 16 \\
\hline VI & Partial substitution & $\mathbf{0 . 8 8 4}$ & $\underline{0.898}$ & $\mathbf{0 . 8 7 0}$ & $\underline{0.900}$ & $\mathbf{0 . 8 2 5}$ & $\underline{0.922}$ & 78 \\
\hline
\end{tabular}

TABLE IV

RESUlts OF EXPERIMENTS WiTH THE POTSDAM DATA SET. THE THREE LEFT COLUMNS ARE AVERAGE VALUES OVER ALL CLASSES, THE RIGHT THREE COLUMNS GIVE PER CLASS $F_{1}$-SCORES. BEST RESULTS ACROSS ALL VARIANTS ARE WRITTEN IN BOLD FONT, SECOND BEST RESULTS ARE UNDERLINED, AND third best results HAVE ITALIC FOND TYPE. ALL EXPERIMENTS (AND RUN-TIMES) WERE COMPUTED ON A STANDARD, STAND-ALONE PC WITH I7 CPU, 2.7 GHz, 64 GB RAM AND NVIDIA TITAN-X GPU WITH 12 GB RAM. LIKE IN TAB. [II] P IS SHORT FOR POTSDAM WHEREAS B IS SHORT FOR BERLIN. 
global" semantic segmentation model we perform a further experiment, where we avoid domain-adaption altogether. The FCN is trained on all Google aerial images plus OSM ground truth (Chicago, Paris, Zurich, Berlin, Potsdam), and then used to predict from the ISPRS images. This achieves significantly worse results ( $0.645 F_{1}$-score). A small set of images with similar radiometry is needed to adapt the classifier to the sensor properties and lighting conditions of the test set.

Finally, we respond to the questions we raised at the beginning of this section. A general consensus is that complete substitution of manually acquired labels achieves acceptable results. Semantic segmentation of overhead images can indeed be learned from OSM maps without any manual labeling effort albeit at the cost of reduced segmentation accuracy. Augmentation of manually labeled training data at very large scale reaches the best overall results. Pre-training with largescale OSM data and publicly available images does improve segmentation of a project-specific data set of independently acquired images and labels (although only by a small margin in this case). An interesting result is that large-scale pre-training on (inaccurate) data increases recall significantly whereas precision slightly drops (compare II and $\mathbf{V}$ in Table IV). Partial substitution of manually labeled training data with large-scale but inaccurate, publicly available data works very well and seems to be a good trade-off between manual labeling effort and segmentation performance. Indeed, pre-training with large-scale OSM labels can replace the vast majority of manual labels. A generic model learned from OSM data adapts very well to a specific location and data source with only little dedicated training data.

\section{CONCLUSIONS AND OUTLOOK}

Traditionally, semantic segmentation of aerial and satellite images crucially relies on manually labelled images as training data. Generating such training data for a new project is costly and time-consuming, and presents a bottleneck for automatic image analysis. The advent of powerful, but data-hungry deep learning methods aggravates that situation. Here, we have explored a possible solution, namely to exploit existing data, in our case open image and map data from the internet for supervised learning with deep CNNs. Such training data is available in much larger quantities, but "weaker" in the sense that the images are not representative of the test images' radiometry, and labels automatically generated from external maps are noisier than dedicated ground truth annotations.

We have conducted a number of experiments that validate our hypothesis stated in the introduction of this paper: (i) the sheer volume of training data can (largely) compensate for lower accuracy, (ii) the large variety present in very large training sets spanning multiple different cities does improve the classifier's ability to generalize to new, unseen locations (see predictions on Tokyo, Fig. 6), (iii) even if high-quality training data is available, the large volume of additional training data improves classification, (iv) large-scale (but lowaccuracy) training data allows substitution of the large majority ( $85 \%$ in our case) of the manually annotated high-quality data.

In summary, we can state that weakly labelled training data, when used at large scale, nevertheless significantly improves segmentation performance, and improves generalization ability of the models. We found that even training only on open data, without any manual labelling, achieves reasonable (albeit far from optimal) results, if the train/test images are from the same source. Large-scale pre-training with OSM labels and publicly available aerial images, followed by domain adaptation to tune to the images at hand, significantly benefits semantic segmentation and should be used as standard practice, as long as suitable images and map data are available.

Online map data, as used in our study, is presently limited to RGB orthophotos with unknown radiometric calibration and street map data for navigation purposes. But we are convinced that comparable training databases can be generated automatically for many problems of interest on the basis of the image and map archives of mapping agencies and satellite data providers. In fact, we are already observing a trend towards free and open data (e.g., the Landsat and MODIS archives, open geodata initiatives from several national mapping agencies, etc.).

At first glance, it seems that object classes with complex contextual relations, like our road class, benefit most from more training data. This intuitively makes sense, because more data is needed to learn complex long-range layout constraints from data, but more research is needed to verify and understand the effects in detail. Moreover, more studies are needed with different class nomenclatures, and more diverse datasets, covering different object scales and image resolutions. A visionary goal would be a large, free, publicly available "model zoo" of pre-trained classifiers for the most important remote sensing applications, from which users world-wide can download suitable models and either apply them directly to their region of interest, or use them as initialization for their own training.

\section{REFERENCES}

[1] M. Haklay and P. Weber, "OpenStreetMap: UserGenerated Street Maps," IEEE Pervasive Computing, vol. 7, no. 4, pp. 12-18, 2008.

[2] M. Haklay, "How Good is Volunteered Geographical Information? A Comparative Study of OpenStreetMap and Ordnance Survey Datasets," Environment and Planning B: Urban Analytics and City Science, vol. 37, no. 4, pp. 682-703, 2010.

[3] J.-F. Girres and G. Touya, "Quality Assessment of the French OpenStreetMap Dataset," Transactions in GIS, vol. 14, no. 4, pp. 435-459, 2010.

[4] J. D. Wegner, S. Branson, D. Hall, K. Schindler, and P. Perona, "Cataloging Public Objects Using Aerial and Street-Level Images Urban Trees," in Computer Vision and Pattern Recognition (CVPR), 2016.

[5] J. Long, E. Shelhamer, and T. Darrell, "Fully convolutional networks for semantic segmentation," in $C V P R$, 2015.

[6] F. Rottensteiner, G. Sohn, M. Gerke, J. D. Wegner, U. Breitkopf, and J. Jung, "Results of the isprs benchmark on urban object detection and $3 \mathrm{~d}$ building reconstruction," ISPRS Journal of Photogrammetry and Remote Sensing, vol. 93, no. 0, pp. 256-271, 2014. 
[7] P. Fua and A. J. Hanson, "Using generic geometric models for intelligent shape extraction," in Proceedings of the Sixth National Conference on Artificial Intelligence, 1987, pp. 706-709.

[8] R. Mohan and R. Nevatia, "Using perceptual organization to extract 3d structures," Transactions on Pattern Analysis and Machine Intelligence, vol. 11, no. 11, pp. 1121-1139, 1989.

[9] M. Herman and T. Kanade, "The 3d mosaic scene understanding system: Incremental reconstruction of $3 \mathrm{~d}$ scenes from complex image," in Image Understanding Workshop, 1984, pp. 137-148.

[10] U. Weidner, "Digital surface models for building extraction," in Automatic Extraction of Man-Made Objects from Aerial and Space Images (II), 1997, pp. 193-202.

[11] A. Fischer, T. H. Kolbe, F. Lang, A. B. Cremers, W. Förstner, L. Plümer, and V. Steinhage, "Extracting buildings from aerial images using hierarchical aggregation in $2 \mathrm{~d}$ and 3d," Computer Vision and Image Understanding, vol. 72, no. 2, pp. 185-203, 1998.

[12] M. Fischler, J. Tenenbaum, and H. Wolf, "Detection of roads and linear structures in low-resolution aerial imagery using a multisource knowledge integration technique," Computer Graphics and Image Processing, vol. 15, pp. $201-223,1981$.

[13] U. Stilla, "Map-aided structural analysis of aerial images," ISPRS Journal of Photogrammetry and Remote Sensing, vol. 50, no. 4, pp. 3-10, 1995.

[14] C. Steger, C. Glock, W. Eckstein, H. Mayer, and B. Radig, "Model-based road extraction from images," in Automatic Extraction of Man-Made Objects from Aerial and Space Images, Birkh auser Verlag Basel. Birkhauser Verlag, 1995, pp. 275-284.

[15] T. Leung and J. Malik, "Representing and Recognizing the Visual Appearance of Materials using Threedimensional Textons," International Journal of Computer Vision, vol. 43, no. 1, pp. 29-44, 2001.

[16] C. Schmid, "Constructing Models for Content-based Image Retrieval," in IEEE Conference on Computer Vision and Pattern Recognition, 2001.

[17] J. Shotton, J. Winn, C. Rother, and A. Criminisi, "Textonboost for image understanding: Multi-class object recognition and segmentation by jointly modeling texture, layout, and context," International Journal of Computer Vision, vol. 81, pp. 2-23, 2009.

[18] P. Viola and M. Jones, "Rapid object detection using a boosted cascade of simple features," in IEEE Conference on Computer Vision and Pattern Recognition, 2001.

[19] P. Dollár, Z. Tu, P. Perona, and S. Belongie, "Integral channel features," in British Machine Vision Conference, 2009.

[20] B. Fröhlich, E. Bach, I. Walde, S. Hese, C. Schmullius, and J. Denzler, "Land cover classification of satellite images using contextual information," ISPRS Annals of the Photogrammetry, Remote Sensing and Spatial Information Sciences, vol. II(3/W1), pp. 1-6, 2013.

[21] P. Tokarczyk, J. D. Wegner, S. Walk, and K. Schindler, "Features, color spaces, and boosting: New insights on semantic classification of remote sensing images," IEEE Transactions on Geoscience and Remote Sensing, vol. 53, no. 1, pp. 280-295, 2015.

[22] R. Stoica, X. Descombes, and J. Zerubia, "A Gibbs Point Process for road extraction from remotely sensed images," IJCV, vol. 57, no. 2, pp. 121 - 136, 2004.

[23] D. Chai, W. Förstner, and F. Lafarge, "Recovering linenetworks in images by junction-point processes," in Computer Vision and Pattern Recognition (CVPR), 2013.

[24] M. Ortner, X. Descombes, and J. Zerubia, "Building Outline Extraction from Digital Elevation Models Using Marked Point Processes," IJCV, vol. 72, no. 2, pp. 107 $-132,2007$.

[25] Y. Verdié and F. Lafarge, "Detecting parametric objects in large scenes by Monte Carlo sampling," IJCV, vol. 106, pp. $57-75,2014$.

[26] S. Kluckner, T. Mauthner, P. M. Roth, and H. Bischof, "Semantic classification in aerial imagery by integrating appearance and height information," in ACCV, 2009.

[27] S. Kluckner and H. Bischof, "Image-based building classification and 3D modeling with super-pixels," in International Archives of Photogrammetry, Remote Sensing and Spatial Information Sciences, vol. 38(3A), 2010, pp. $233-238$.

[28] J. D. Wegner, J. Montoya, and K. Schindler, "A higherorder crf model for road network extraction," in Computer Vision and Pattern Recognition (CVPR), 2013.

[29] J. Montoya, J. D. Wegner, L. Ladicky, and K. Schindler, "Mind the gap: modeling local and global context in (road) networks," in German Conference on Pattern Recognition, 2014.

[30] J. D. Wegner, J. Montoya, and K. Schindler, "Road networks as collections of minimum cost paths," ISPRS Journal of Photogrammetry and Remote Sensing, vol. 108, pp. 128-137, 2015.

[31] M. Herold, X. Liu, and K. C. Clarke, "Spatial metrics and image texture for mapping urban land use," Photogrammetric Engineering and Remote Sensing, vol. 69, no. 9, pp. 991-1001, 2003.

[32] M. Dalla Mura, J. Benediktsson, B. Waske, and L. Bruzzone, "Morphological attribute profiles for the analysis of very high resolution images," IEEE TGRS, vol. 48, no. 10, pp. 3747-3762, 2010.

[33] W. Schwartz, A. Kembhavi, D. Harwood, and L. Davis, "Human detection using partial least squares analysis," in IEEE International Conference on Computer Vision, 2009.

[34] S. Hussain and B. Triggs, "Feature Sets and Dimensionality Reduction for Visual Object Detection," in British Machine Vision Conference, 2010.

[35] F. van Coillie, L. Verbeke, and R. D. Wulf, "Feature selection by genetic algorithms in object-based classification of IKONOS imagery for forest mapping in Flanders, Belgium," Remote Sensing of Environment, vol. 110, pp. 476-487, 2007.

[36] Y. Rezaei, M. Mobasheri, M. V. Zoej, and M. Schaepman, "Endmember Extraction Using a Combination of Orthogonal Projection and Genetic Algorithm," IEEE 
Geoscience and Remote Sensing Letters, vol. 9, no. 2, pp. 161-165, 2012.

[37] K. Fukushima, "Neocognitron: A self-organizing neural network model for a mechanism of pattern recognition unaffected by shift in position," Biological Cybernetics, vol. 36, no. 4, pp. 193-202, 1980.

[38] Y. LeCun, B. Boser, J. S. Denker, D. Henderson, R. E. Howard, W. Hubbard, and L. D. Jackel, "Backpropagation applied to handwritten zip code recognition," Neural Computation, vol. 1, no. 4, pp. 541-551, 1989.

[39] O. Russakovsky, J. Deng, H. Su, J. Krause, S. Satheesh, S. Ma, Z. Huang, A. Karpathy, A. Khosla, M. Bernstein, A. Berg, and L. Fei-Fei, "Imagenet Large Scale Visual Recognition Challenge," International Journal of Computer Vision, vol. 115, no. 3, pp. 211-252, 2015.

[40] A. Krizhevsky, I. Sutskever, and G. E. Hinton, "Imagenet classification with deep convolutional neural networks," in Advances in Neural Information Processing Systems (NIPS), 2012.

[41] K. Simonyan and A. Zisserman, "Very deep convolutional networks for large-scale image recognition," in International Conference on Learning Representations (ICLR), 2015.

[42] C. Farabet, C. Couprie, L. Najman, and Y. LeCun, "Learning Hierarchical Features for Scene Labeling," IEEE Transactions on Pattern Analysis and Machine Intelligence, 2013, to appear, available online.

[43] S. Paisitkriangkrai, J. Sherrah, P. Janney, and A. van den Hengel, "Effective semantic pixel labelling with convolutional networks and conditional random fields," in CVPR Workshops, Computer Vision and Pattern Recognition, 2015.

[44] A. Lagrange, B. Le Saux, A. Beaupere, A. Boulch, A. Chan-Hon-Tong, S. Herbin, H. Randrianarivo, and M. Ferecatu, "Benchmarking classification of earthobservation data: from learning explicit features to convolutional networks," in International Geoscience and Remote Sensing Symposium (IGARSS), 2015.

[45] D. Marmanis, K. Schindler, J. D. Wegner, and S. Galliani, "Semantic segmentation of aerial images with an ensemble of cnns," ISPRS Annals - ISPRS Congress, 2016.

[46] V. Mnih and G. E. Hinton, "Learning to detect roads in high-resolution aerial images," in European Conference on Computer Vision, 2010.

[47] _ - "Learning to label aerial images from noisy data," in International Conference on Machine Learning, 2012.

[48] G. Máttyus, S. W. anf Sanja Fidler, and R. Urtasun, "Enhancing road maps by parsing aerial images around the world," in International Computer Vision Conference, 2015, pp. 1689-1697.

[49] V. Mnih, "Machine learning for aerial image labeling," Ph.D. dissertation, University of Toronto, 2013.

[50] E. Maggiori, Y. Tarabalka, G. Charpiat, and P. Alliez, "Convolutional Neural Networks for Large-Scale Remote Sensing Image Classification," Transactions on Geoscience and Remote Sensing, vol. 55, no. 2, pp. 645-657, 2017.

[51] V. Nair and G. E. Hinton, "Rectified linear units improve restricted boltzmann machines," in International Conference on Machine Learning (ICML), 2010.

[52] X. Glorot and Y. Bengio, "Understanding the difficulty of training deep feedforward neural networks," in International Conference on artificial intelligence and statistics, 2010, pp. 249-359.

[53] M. Everingham, L. Van Gool, C. K. Williams, J. Winn, and A. Zisserman, "The Pascal visual object classes (VOC) challenge," International Journal of Computer Vision, vol. 88, no. 2, pp. 303-338, 2010.

[54] F. Rottensteiner, G. Sohn, M. Gerke, and J. D. Wegner, "ISPRS Test Project on Urban Classification and 3D Building Reconstruction," ISPRS Working Group III / 4 - 3D Scene Analysis, Tech. Rep., 122013. [Online]. Available: http://www2.isprs.org/tl_files/isprs/ wg34/docs/ComplexScenes_revision_v4.pdf

[55] E. Maggiori, G. Charpiat, Y. Tarabalka, and P. Alliez, "Learning Iterative Processes with Recurrent Neural Networks to Correct Satellite Image Classification Maps," arXiv preprint arXiv:1608.03440v2, 2016. 